\title{
LE DUAL DES FONCTIONS HOLOMORPHES INTÉGRABLES SUR UN DOMAINE STRICTEMENT PSEUDO-CONVEXE
}

\author{
BERNARD COUPET
}

(Communicated by Irwin Kra)

\begin{abstract}
We prove that on a strictly pseudoconvex domain with $C^{4}$ boundary in $C^{\prime \prime}$, the dual of the Bergman space $B^{1}$ is Bloch space.
\end{abstract}

Nous démontrons que le dual de l'espace des fonctions holomorphes intégrables sur un domaine strictement pseudo-convexe de classe $C^{4}$ s'identifie à l'espace des fonctions holomorphes dont le gradient est controlé par l'inverse de la distance au bord.

$D$ désigne un domaine borné de $\mathbf{C}^{n}$ défini par une fonction $\rho$ de classe $C^{2}$ dont le gradient ne s'annule pas sur le bord. $\tilde{B}$ est l'ensemble des fonctions $f$ différentiables sur $D$ telles que $\operatorname{Sup}_{z \in D}|\rho(z) \nabla f(z)|<+\infty$. Pour tout réel $\varepsilon>0$, on pose $D_{\varepsilon}=$ $\rho^{-1}(]-\infty,-\varepsilon[)$ et $V_{\varepsilon}=\rho^{-1}(]-\varepsilon, \varepsilon[)$. D étant $C^{2}$, il existe $C$ et $\varepsilon_{0}$ tel que pour $z_{0}$ et $z_{1}$ dans $V_{\varepsilon_{0}}$ il existe un chemin differentiable joignant $z_{0}$ et $z_{1}$ et de longueur majorée $C\left|z_{0}-z_{1}\right|$.

Pour tout $f$ de $\tilde{\mathscr{B}}$ on pose

$$
\|f\|_{\tilde{B}}=\operatorname{Sup}_{D \mid V_{\varepsilon_{0}}}|f|+\operatorname{Sup}_{D}|\rho(z) \nabla f(z)| .
$$

$\tilde{\mathscr{B}}$ muni de cette norme est un espace de Banach. L'espace de Bloch $\mathscr{B}$ est $H(D) \cap \tilde{\mathscr{B}}$. Un élément de $\mathscr{B}$ est un $O(\log -1 / \rho)$. L'espace de Bergman $B^{p}$ est l'ensemble des fonctions holomorphes sur $D$ qui sont aussi dans $L^{p}$; on peut remarquer que $\mathscr{B}$ est inclus dans $B^{p}$ pour tout réel $p$.

La projection orthogonale de $L^{2}$ sur $B^{2}$, notée $P$, est appelée la projection de Bergman. $C$ 'est un opérateur intégral dont le noyau sera noté $K$. Pour tout $w$ dans $D, K(\cdot, w)$ est holomorphe et $K(w, \cdot)$ est antiholomorphe sur $D$.

Dans le cas de disque unité, il est bien connu que le dual de $B^{1}$ est $\mathscr{B}$, la dualité étant donnée par celle de $L^{2}$; nous généralisons cela pour les domaines strictement pseudo-convexe de classe $C^{4}$. Dans une première partie, nous démontrerons que $B^{1}$ et $\mathscr{B}$ sont en dualité pour tout domaine $D$ de classe $C^{2}$. Dans une seconde partie, nous verrons que dans le cas des domaines strictement pseudo-convexes, cette dualité permet d'identifier $\left(B^{1}\right)^{*}$ et $\mathscr{B}$. Pour terminer, nous étudierons le cas de domaines produits.

Received by the editors August 11, 1986. This paper was presented at "Journèes compleses du Sud" meetings in Marseille (France) May 10, 1986.

1980 Mathematics Subject Classification (1985 Revision). Primary 46E15; Secondary 32H10. 
I. Dualité de $B^{1}$ et $\mathscr{B}$. Cette dualité découle des lemmes suivants:

LeMme 1. Toute fonction $U$ de la forme $\partial(\theta \rho) / \partial z_{j}$ où $\theta$ est de classe $C^{1}$ sur $D$ et vérifie l'estimation

$$
\nabla \theta=O(-1 / \rho)
$$

est orthogonale à $B^{2}$.

Demonstration. Commençons par traiter le cas où $\theta$ est à support compact inclus dans $D$. Soit $f$ dans $B^{2}$.

$$
\begin{aligned}
(U \mid f) & =\int_{D} \overline{f(w)} U(x) \bigwedge_{k=1}^{n}\left(d w_{k} \wedge d \bar{w}_{k}\right) \\
& =\int_{D} d\left[\overline{f(w)} \theta(w) \rho(w) d \bar{w}_{j} \wedge\left(\bigwedge_{k \neq j}\left(d w_{k} \wedge d \bar{w}_{k}\right)\right)\right] .
\end{aligned}
$$

Par le théorème de Stokes cette dernière intégrale est nulle car la forme à intégrer est à support compact dans $\Omega$.

Le lemme va découler de ce cas particulier par densité.

Soit $\left(\varphi_{m}\right)$ une suite de fonction $C^{1}$ sur $C^{n}$ vérifiant

$$
\begin{aligned}
\varphi_{m} & =1 \quad \operatorname{sur} V_{2^{-m}}, \\
\operatorname{Supp} \varphi_{m} & \subset V_{2^{-m+1}}, \\
\left|\nabla \varphi_{m}\right| & \leqslant C 2^{m} .
\end{aligned}
$$

Posons $\chi_{m}=1-\varphi_{m}$ la suite $U_{m}=\partial\left(\theta \rho X_{m}\right) / \partial z_{j}$ converge vers $U$ dans $L^{2}$.

Puisque $U_{m} \chi_{m} U+\theta \rho \partial \chi_{m} / \partial z_{j}$ et $U=\lim _{m \rightarrow \infty} \chi_{m} U$ dans $L^{2}$ il est suffisant de vérifier que $\theta \rho \partial X_{m} / \partial z_{j}$ converge vers 0 dans $L^{2}$.

Or

$$
\int_{D}\left|\theta \rho \frac{\partial \chi_{m}}{\partial z_{j}}\right|^{2}=O\left(m^{2} \operatorname{mes}\left(V_{2^{m-1}}\right)\right)=O\left(m^{2} 2^{-(m-1)}\right) .
$$

LEMME 2. Il existe un opérateur continu $L$ de $\tilde{\mathscr{B}}$ dans $L^{\infty}$ tel que $P L=P$.

Démonstration. Considérons des ouverts $\left(O_{j}\right)_{1 \leqslant j \leqslant n}$ dont la réunion recouvre $b D$ et tels que sur chaque $O_{j} \partial \rho / \partial z_{j}$ soit minoré par une constante $C$ et soit $\left(\alpha_{j}\right)_{1 \leqslant j \leqslant n}$ une partition de l'unité subordonnée a ce recouvrement [5].

L'opérateur $L$ est défini par:

$$
L(g)=g+\sum_{j=1}^{n} \frac{\partial}{\partial z_{j}}\left(-\frac{\rho g \cdot \alpha_{j}}{\partial \rho / \partial z_{j}}\right) .
$$

D'après le lemme 1, le second terme est orthogonal à $B^{2}$; par suite $P L(g)=P(g)$. $L(g)$ s'écrit encore comme somme de trois termes $L_{1}+L_{2}+L_{3}$ :

$$
\begin{aligned}
L(g)= & g\left(1-\sum_{j=1}^{n} \alpha_{j}\right)+\sum_{j=1}^{n} \frac{-\rho}{\partial \rho / \partial z_{j}} \frac{\partial\left(\alpha_{j} g\right)}{\partial z_{j}} \\
& +\rho g \sum_{j=1}^{n} \frac{\alpha_{j}}{\left(\partial \rho / \partial z_{j}\right)^{2}} \frac{\partial^{2} \rho}{\partial z_{j}^{2}} .
\end{aligned}
$$


La fonction $1-\sum_{j=1}^{n} \alpha_{j}$ étant nulle au voisinage de $b D$, le premier terme est majoré par $\operatorname{Sup}_{D \mid Q O_{i}}|g|$ soit par $\|g\|_{\tilde{\mathscr{B}}}$.

Chaque terme dans la somme $L_{2}$ se majore de la façon suivante:

où $A$ est indépendante de $g$.

$$
\begin{aligned}
\left|\frac{-\rho}{\partial \rho / \partial z_{j}} \frac{\partial\left(g \alpha_{j}\right)}{\partial z_{j}}\right| & \leqslant \frac{1}{C}\left|\rho \frac{\partial g}{\partial z_{j}} \alpha_{j}\right|+\frac{1}{C}|\rho g|\left|\frac{\partial \alpha_{j}}{\partial z_{j}}\right| \\
& \leqslant \frac{1}{C}\|g\|_{\mathscr{B}}+\frac{1}{C}\left|\rho \log \frac{-1}{\rho}\right|\|g\|_{\mathscr{B}}\left|\frac{\partial \alpha_{j}}{\partial z_{j}}\right| \\
& \leqslant A\|g\|_{\mathscr{B}}
\end{aligned}
$$

La somme dans $L_{3}$ est indépendante de $g$ et est fonction continue à support compact donc bornée. Par la suite le troisième terme est majoré par $A|\rho g|$ donc par $A\|g\|_{\tilde{\mathscr{B}}}$.

Il en découle que $L(g)$ appartient à $L^{\infty}$ et que $L$ est continu.

Voici le lemme fondamental.

LEMME 3. Il existe une constante $K$ telle que pour toute fonction $f$ de $B^{2}$ et tout $g$ de $\mathscr{B}$,

$$
\left|\int_{D} f \cdot \bar{g}\right| \leqslant K\|f\|_{1}\|g\|_{\mathscr{B}}
$$

En effet:

$$
\begin{aligned}
\int_{D} f \bar{g} & =\int_{D} f \cdot \overline{P L(g)} \quad \text { car } P L(g)=g \\
& =\int_{D} P(f) \overline{L(g)} \quad \text { car } P \text { est autoadjoint } \\
& =\int_{D} f \overline{L(g)} \quad \text { car } f \text { est holomorphe. }
\end{aligned}
$$

Il suffit maintenant d'appliquer le lemme 2.

THEORÈmE 1. La forme

$$
B^{2} \times \mathscr{B} \rightarrow \mathbf{C}, \quad(f, g) \mapsto \int_{D} f \bar{g},
$$

se prolonge à $B^{1} \times \mathscr{B}$ en une forme continue.

$\dot{A} g$ dans $\mathscr{B}$ associons la forme linéaire $l_{g}$ sur $B^{1}$ définie par

$$
l_{g}(l)=\int_{D} f \cdot \overline{L(g)} .
$$

$D$ 'après le lemme $2, l_{g}$ est continue et $\left\|l_{g}\right\| \leqslant K\|g\|_{\mathscr{B}}$ et si f est dans $B^{2}, l_{g}(f)=$ $\int_{D} f \cdot \bar{g}$.

L'application $g \mapsto l_{g}$ est injective de $\mathscr{B}$ dans $\left(B^{1}\right)^{*}$. En effet si $l_{g}=0,0=l_{g}(g)=$ $\int|g|^{2}$ donc $g=0$.

II. Isomorphisme entre $\left(B^{1}\right)^{*}$ et $\mathscr{B}$. Notons, pour $f$ dans $B^{1}$ et $g$ dans $\mathscr{B},(f \mid g)$ l'intégrale $\int_{D} f \cdot \overline{L(g)}$. Nous étudions maintenant si cette dualité permet de définir un isomorphisme entre $\left(B^{1}\right)^{*}$ et $\mathscr{B}$, ce que nous écrirons $\left(B^{1}\right)^{*}=\mathscr{B}$. Nous allons 
donner une condition nécessaire et suffisante pour que cela soit réalisé et démontrer que cette condition est vérifiée lorsque $D$ est un domaine strictement pseudoconvexe de classe $C^{4}$. Nous donnerons aussi un exemple où cette condition est en défaut.

Proposition 1. $\left(B^{1}\right)^{*}=\mathscr{B}$ si et seulement si les deux conditions suivantes sont vérifiées:

(1) $B^{2}$ est dense dans $B^{1}$.

(2) $P\left(L^{\infty}\right) \subset \mathscr{B}$.

Demonstration. Supposons pour commencer $\left(B^{1}\right)^{*}=\mathscr{B}$. Par le théorème de Hahn-Banach, pour vérifier que $B^{2}$ est dense dans $B^{1}$, il suffit de démontrer qu'une forme, linéaire $l$ nulle sur $B^{2}$ est nulle. Soit $h$ appartenant à $\mathscr{B}$ telle que:

$$
\forall f \in B^{1} \quad l(f) \asymp \int_{D} f \cdot \overline{L(h)} .
$$

En particulier pour tout $f$ de $B^{2} \int_{D} f \cdot \overline{L(h)}=0$ et donc $P L(h)=0$ et donc $h=0$ et $l=0$. Soit $g$ un élément de $L^{\infty}$. Par hypothèse, il existe $h \in \mathscr{B}$ tel que pour tout $f \operatorname{de~} B^{1} \int_{D} f \cdot \overline{L(h)}=\int_{D} f \cdot \bar{g}$ et donc pour tout $f \operatorname{de~} B^{2} \int_{D} f(\overline{L(h)}-g)=0$, c'est-à-dire $P L(h)=P(g)$ soit $P(g)=h$ et $P(g)$ appartient à $\mathscr{B}$. Supposons que les deux conditions soient réalisées.

Si $l$ est une forme linéaire continue sur $B^{1}$, par le théorème de Hahn-Banach, il existe $g$ appartenant à $L^{\infty}$ tel que:

$$
\forall f \in B^{1} \quad l(f)=\int_{D} f \cdot \bar{g} .
$$

En particulier pour tout $f$ de $B^{2}$

$$
l(f)=\int_{D} f \cdot \overline{P(g)}=(f \mid P(g))
$$

car $P(g)$ appartient à $\mathscr{B}$ par hypothèse. Par densité de $B^{2}$ dans $B^{1}$ on conclut que $l$ est représentée par l'élément $P(g)$ de $\mathscr{B}$.

Proposition 2. L'inclusion $P\left(L^{\infty}\right) \subset \mathscr{B}$ est vérifiée si et seulement si le noyau de Bergman vérifie l'estimation

$$
\operatorname{Sup}_{1 \leqslant j \leqslant n} \sup _{w \in \Omega}|\rho(z)| \int_{D}\left|\frac{\partial}{\partial z_{j}} K(z, w)\right| d \lambda(w)<+\infty .
$$

Il est immédiat que cette condition assure $P\left(L^{\infty}\right) \subset \mathscr{B}$. Inversement si $P\left(L^{\infty}\right) \subset \mathscr{B}$, on a en fait $P\left(L^{\infty}\right)=\mathscr{B}$ par le lemme 2 et donc par le théorème du graphe fermé $P$ est continu de $L^{\infty}$ sur $\mathscr{B}$, c'est à dire qu'il existe une constante $c>0$ telle que:

$$
\forall g \in L^{\infty} \quad\|P(g)\|_{\mathscr{B}} \leqslant C\|g\|_{\infty} .
$$

En particulier pour tout $g \in C_{0}(D)$ on obtient, pour tout $j$ :

$$
|\rho(z)|\left|\int \frac{\partial K}{\partial z_{j}}(z, w) g(w) d \lambda(w)\right| \leqslant C\|g\|_{\infty}
$$


et donc par dualité entre $C_{0}$ et $L^{1}$

$$
|\rho(z)| \int\left|\frac{\partial K}{\partial z_{j}}(z, w)\right| d \lambda(w) \leqslant C .
$$

Proposition 3. Il existe un domaine $D$ de $\mathbf{C}^{2}$ de classe $C^{\infty}$ tel qu'aucune des deux conditions de la proposition précédente ne soit vérifiée.

Il suffit de reprendre le domaine $D$ construit dans [1], la condition 2 n'est pas vérifiée puisque $P\left(C_{0}^{\infty}\right)$ n'est pas inclus dans $L^{p}$ (pour $p \geqslant 4$ ).

La vérification du fait que la condition 1 n'est pas vérifiée se traite en reprenant la démonstration du théorème et du lemme 3 de [1] avec la fonction $1 / z^{2}$ qui appartient à $B^{1}$. Voici une indication:

- S'il existe une suite $\left(g_{j}\right)$ d'éléments de $B^{2}$ approchant $1 / z^{2}$ dans $B^{1}$, il existe une suite $\left(h_{j}\right)$ d'éléments de $B^{2}$ approchant $1 / z^{2}$ dans $L^{1}$ et indépendantes de la variable $w$.

-Un élément $h$ indépendant de la variable $w$ appartient à $B^{2}$ si et seulement si il est de la forme $a / z+g$ avec $g$ holomorphe sur le disque du plan $\{|z|<4\}$.

$-\mathrm{Si}\left(h_{j}\right)$ tend vers $1 / z^{2}$ dans $L^{1}(D), h_{j}$ tens vers $1 / z^{2}$ dans $L^{1}\left(D_{r}\right)$ pour $1<r<2$ où $D_{r}$ est une "couronne" contenant le cercle $|z|=3$.

-Il $y$ a contradiction, puisque les coefficients du développement de Laurent d'une fonction holomorphe sont continus en norme $L^{1}$.

Nous démontrons maintenant:

THEORÈmE 2. Si D est un domaine strictement pseudo-convexe de classe $C^{4}$, $\left(B^{1}\right)^{*}=\mathscr{B}$.

Demonstration. Puisque $B^{2}$ est dense dans $B^{1}$, il s'agit de démontrer l'estimation

$$
\operatorname{Sup}_{z}|\rho(z)| \int\left|\frac{\partial K}{\partial z_{j}}(z, w) d \lambda(w)\right|<+\infty .
$$

Dans les cas des domaines $C^{\infty}$ on peut appliquer le développement du noyau de Bergman de [2]. Pour les domaines qui sont seulement $C^{4}$ nous reprenons la méthode de [4].

L'idée est de construire une projection explicite $G$ de $L^{2}$ sur $B^{2}$ et de démontrer que $G$ opère de $L^{\infty}$ dans $\mathscr{B}$ et que $\left[I-\left(G^{*}-G\right)\right]^{1}$ opère sur $L^{\infty}$. Cela implique que $P=G\left(I+G-G^{*}\right)^{-1}$ opère de $L^{\infty}$ dans $\mathscr{B}$.

Rappelons brièvement la construction faite par E. Ligocka dans [4]. Soit $\rho$ une fonction strictement pluri-sous harmonique dans un voisinage de $\bar{D}$ de classe $C^{4}$. Soit $L \rho$ est la forme de Levi, on peut trouver des réels $\varepsilon_{0}$ et $\delta_{0}$ tels que

$$
L \rho(z)(z-w) \geqslant C|z-w|^{2} \text { si }|\rho(z)|<\delta_{0} \text { et }|z-w|<\varepsilon_{0} .
$$

On pose

$$
F_{1}(z, w)=\sum_{j=1}^{n} \frac{\partial \rho}{\partial z_{j}}(w)\left(w_{j}-z_{j}\right)+\frac{1}{2} \sum_{j, k} \frac{\partial^{2} \rho}{\partial z_{j} \partial z_{k}}\left(z_{j}-w_{j}\right)\left(z_{k}-w_{k}\right)
$$


et

$$
F(z, w)=\psi(t) F_{1}(z, w)+(1-\psi(t))|w-z|^{2}
$$

où $t=|z-w|$ et $\psi$ est une fonction de troncature valant 1 si $t<\varepsilon_{0} / 4$ et 0 si $t>\varepsilon_{0} / 2$.

Le noyau $G$ s'écrit

$$
\frac{A(z, w)}{[F(z, w)-\rho(w)]^{n+1}}
$$

où $A$ est $C^{\infty} \times C^{1}$ sur $\bar{D} \times \bar{D}$. Les propriétés de la projection $G$ sont:

1. $G$ est une projection continue de $L^{2}$ sur $B^{2}$.

2. $B=G^{*}-G$ est un opérateur continu de $L^{\infty}$ dans $\operatorname{Lip}_{1 / 2}$ et donc $I-B$ est inversible d'inverse continu sur $L^{\infty}$.

3. $G$ opère de $L^{\infty}$ dans $\mathscr{B}$.

Les faits 1 et 2 sont démontrés par E. Ligocka et il reste donc à vérifier l'estimation:

$$
\int_{D}\left|\frac{\partial G}{\partial z_{j}}(z, w)\right| d \lambda(w) \leqslant \frac{C}{|\rho(z)|}
$$

où $C$ est une constante indépendante de $z$. Or

$$
\frac{\partial G}{\partial z_{j}}(z, w)=\frac{A_{j}(z, w)}{[F(z, w)-\rho(w)]^{n+2}}
$$

où $A_{j}$ est une fonction continue sur $\bar{D} \times \bar{D}$ donc bornée. Il suffit donc de démontrer:

$$
\int_{D} \frac{d \lambda|w|}{|F(z, w)-\rho(w)|^{n+2}} \leqslant \frac{C}{|\rho(z)|},
$$

$\rho$ étant strictement pluri-sous harmonique, il est classique que

$$
\operatorname{Re} F(z, w)-\rho(w) \geqslant C\left[-\rho(z)-\rho(w)+|z-w|^{2}\right]
$$

pour $z$ appartenant à $D$ et $w$ à $\bar{D}$. Il est donc suffisant de démontrer:

$$
\int_{D} \frac{d \lambda(w)}{\left[-\rho(z)-\rho(w)+|z-w|^{2}\right]^{n+2}} \leqslant \frac{C}{|\rho(z)|},
$$

ce qui est connu [3].

Proposition 4. $S i\left(B^{1}\right)^{*}=\mathscr{B}$, il existe une projection continue de $L^{1}$ sur $B^{1}$.

DEmonstration. Nous commençons par démontrer la formule:

$$
\forall z \in D \forall f \in B^{1} \quad f(z)=\int_{D} \overline{L_{w} K(w, z)} f(w) d \lambda(w) .
$$

Fixons $z$ dans $D$. La forme linéaire $\delta_{z}: f \mapsto f(z)$ étant continue sur $B^{1}$, il existe, puisque $\left(B^{1}\right)^{*}=\mathscr{B}, \varphi_{z}$ appartenant à $\mathscr{B}$ telle que:

$$
\forall f \in B^{1} \quad f(z)=\int_{D} \overline{L_{w} \varphi_{z}(w)} f(w) d \lambda(w) .
$$


En particulier, pour $f$ dans $B^{2}$, nous en déduisons:

$$
f(z)=\int_{D} \overline{\varphi_{z}(w)} f(w) d \lambda(w) .
$$

$K(\cdot, z)$ étant l'unique élément de $B^{2}$ représentant la forme linéaire $\delta_{z}$ continue sur $B^{2}$, nous obtenons l'égalité:

$$
\forall z \in D \forall w \in D \quad K(w, z)=\varphi_{z}(w)
$$

d'où la formule annoncée.

Posons maintenant $Q(w, z)=\overline{L_{w} K(w, z)}$ et démontrons:

$$
\operatorname{Sup}_{w \in D} \int_{D}|Q(w, z)| d \lambda(z)<+\infty \text {. }
$$

Par définition de $L_{w}$ :

$$
|Q(w, z)| \leqslant[a(w)+|\rho(w)|]|K(w, z)|+C|\rho(w)|\left|\nabla_{w} K(w, z)\right|
$$

où $a$ est une fonction continue à support compact inclus dans $D$. Par suite:

$$
\begin{aligned}
\int_{D}|Q(w, z)| d \lambda(z) \leqslant & {[a(w)+\mid \rho(w)] \int_{D}|K(w, z)| d \lambda(z) } \\
& +C|\rho(w)| \int_{D}\left|\nabla_{w} K(w, z)\right| d \lambda(z) .
\end{aligned}
$$

Comme

$$
\int_{D}\left|\nabla_{w} K(w, z)\right| d \lambda(z) \leqslant \frac{A}{|\rho(w)|}
$$

nous obtenons:

$$
\int_{D}|Q(w, z)| d \lambda(z) \leqslant C\left[A+a(w) \log \frac{1}{|\rho(w)|}+|\rho(w)| \log \frac{1}{|\rho(w)|}\right]
$$

et par suite:

$$
\operatorname{Sup}_{w \in D} \int_{D}|Q(w, z)| d \lambda(z)<+\infty
$$

Définissons pour terminer, l'opérateur $Q \operatorname{sur} L^{1}$ par:

$$
\forall f \in L^{1} \quad Q(f)(z)=\int_{D} Q(w, z) f(w) d \lambda(w) .
$$

D'après ce qui précède $Q$ est un opérateur linéaire continu sur $L^{1}$ reproduisant les éléments de $B^{1} ; Q(f)$ étant aussi holomorphe, la démonstration de la proposition est acheveé.

III. Domaines produits. Nous considérons deux domaines $D_{1}$ et $D_{2}$ de classe $C^{2}$ et leur produit $D=D_{1} \times D_{2}$. Le noyau de Bergman $K$ de $D$ est le produit $K_{1} \otimes K_{2}$ des noyaux de Bergman de $D_{1}$ et $D_{2}$.

Nous définissons l'espace $\mathscr{B}$ comme l'ensemble des fonctions $g$ holomorphes sur $D$ telles que

$$
\operatorname{Sup}_{\left(z^{1}, z^{2}\right) \in D} \rho_{1}\left(z^{1}\right) \rho_{2}\left(z^{2}\right)\left|D_{z_{1}} D_{z_{2}} g\left(z^{1}, z^{2}\right)\right|<+\infty
$$


Comme précédemment la dualité de $B^{1}$ et $\mathscr{B}$ découle de:

Proposition. Il existe un opérateur différentiel $L d$ 'ordre 2 continu de $\mathscr{B}$ dans $L^{\infty}$ tel que $P L=\mathrm{Id}$.

Demonstration. L'opérateur $L$ est le produit des opérateurs $L_{1}$ et $L_{2}$ construits pour $D_{1}$ et $D_{2}$. L agit séparément en chaque variable.

En conservant les notations du lemme 2, $L g-g$ s'écrit

$$
\sum_{j=1}^{n} \frac{\partial}{\partial z_{j}^{1}}\left(-\rho_{1} g \alpha_{j}^{1}\right)+\sum_{k=1}^{m} \frac{\partial}{\partial z_{k}^{2}}\left(-g \rho_{2} \alpha_{k}^{2}\right)+\sum_{j, k} \frac{\partial}{\partial z_{k}^{2}}\left[-\rho \alpha_{k}^{2} \frac{\partial}{\partial z_{j}^{1}}\left(-\rho_{1} g \alpha_{j}^{1}\right)\right] .
$$

Pour vérifier l'orthogonalité de $L g-g$ à $B^{2}(D)$ il suffit, par densité de $B^{2}\left(D_{1}\right) \otimes$ $B^{2}\left(D_{2}\right)$ dans $B^{2}(D)$, de vérifier la nullité de l'intégrale

$$
\int_{D_{1}} \int_{D_{2}} U\left(z^{1}\right) V\left(z^{2}\right) \overline{(L g-g)\left(z^{1}, z^{2}\right)} d \lambda\left(z^{1}\right) d \lambda\left(z^{2}\right)
$$

pour tout $U$ de $B^{2}\left(D_{1}\right)$ et tout $V$ de $B^{2}\left(D_{2}\right)$. Par le lemme 1:

$$
\int_{D_{1}} U \overline{\frac{\partial}{\partial z_{j}^{1}}\left(-\rho_{1} g \alpha_{j}^{1}\right)}
$$

est nul pour tout $z^{\prime}$ de $D^{2}$ et de même

$$
\int_{D_{2}} V \overline{\frac{\partial}{\partial z_{k}^{2}}\left(-\rho_{2} g \alpha_{k}^{2}\right)}
$$

est nul pour tout $z_{1}$ de $D^{1}$. Le troisième terme se décompose sous la forme:

$$
-2 \frac{\partial}{\partial z_{k}^{2}}\left[\alpha_{k}^{2} \frac{\partial}{\partial z_{j}^{1}}\left(-\rho_{1} g \alpha_{j}^{1}\right)\right]+\frac{\partial}{\partial z_{k}^{2}}\left(-\rho_{2}\right) \alpha_{k}^{2} \frac{\partial}{\partial z_{j}^{1}}\left(-\rho_{1} g \alpha_{j}^{1}\right)
$$

et on conclut comme précédemment par le lemme 1 , le premier terme étant orthogonal à $B^{2}\left(D_{2}\right)$ à $z^{1}$ fixé dans $D_{1}$ et le second à $B^{2}\left(D^{1}\right)$ à $z^{2}$ fixé dans $D_{2}$.

Pour vérifier que $L(g)$ est bornée, il suffit de remarquer que $L_{j}(h)$ s'écrit $\rho_{j} a_{j} h+\rho_{j} D_{z^{j}} h\left(z^{j}\right)\left(\nabla \rho_{j}\right)$ où $a_{j}$ est continue au voisinage de $D_{j}$. $L g\left(z^{1} z^{2}\right)$ s'écrit algors:

$$
\begin{aligned}
& \rho_{1}\left(z^{1}\right) \rho_{2}\left(z^{2}\right) a_{1}\left(z^{1}\right) a_{2}\left(z^{2}\right) g\left(z^{1}, z^{2}\right) \\
& \quad+\rho_{2}\left(z^{2}\right) a_{2}\left(z^{2}\right) D_{z^{1}}(g)\left(z^{1}, z^{2}\right)\left(\nabla \rho_{1}\left(z^{1}\right)\right) \\
& \quad+\rho_{1}\left(z^{1}\right) a_{1}\left(z^{1}\right) D_{z^{2}}(g)\left(z^{1}, z^{2}\right)\left(\nabla \rho_{2}\left(z^{2}\right)\right) \\
& \quad+\rho_{1}\left(z^{1}\right) \rho_{2}\left(z^{2}\right) D_{z^{1}} D_{z^{2}} g\left(z^{1}, z^{2}\right)\left(\nabla \rho_{1}\left(z^{1}\right) \nabla \rho_{2}\left(z^{2}\right)\right)
\end{aligned}
$$

ce qui permet de vérifier que $L(g)$ est borné.

On obtient comme précédemment:

ThEorème 3. Soient $D_{1}$ et $D_{2}$ deux domaines strictement pseudo-convexes de classe $C^{4}$. Alors $\mathscr{B}$ s'identifie au dual de $B^{1}$.

\section{REFERENCES}

1. D. Barrett, Irregularity of the Bergman projection on a smooth bounded domain in $\mathbf{C}^{n}$, Ann. of Math. (2) 119 (1984), 431-436.

2. L. Boutet de Monvel and J. Sjöstrand, Sur la singularité des noyaux de Bergman et de Szegö, Astérisque 34-35 (1976), 123-164. 
3. P. Kranz, Optimal Lipschitz and $L^{p}$ regularity for the equations $\bar{\partial} U=f$ on strongly pseudoconvex domains, Math. Ann. 219 (1975), 233-260.

4. E. Ligocka, The Hölder continuity of the Bergman projection and proper holomorphic mappings, Studia Math. 80 (1984), 89-107.

5. S. Bell, Biholomorphic mappings and the $\bar{\partial}$-problem, Ann. of Math. (2) 114 (1981), 103-113.

U. E. R. de Mathematiques et CNRS Ua225, Universite de Provence, 3 Place Victor Hugo, 13331 Marseille Cedex, France 